\title{
ohmage
}

\section{An open Mobile System for Activity and Experience Sampling}

\author{
Nithya Ramanathan, Faisal Alquaddoomi, Hossein Falaki, Dony George, Cheng-Kang Hsieh, John Jenkins, Cameron \\ Ketcham, Brent Longstaff, Jeroen Ooms, Joshua Selsky, Hongsuda Tangmunarunkit, Deborah Estrin \\ \{nithya, destrin\}@ cs.ucla.edu \\ Computer Science Department \\ University of California, Los Angeles \\ Los Angeles, USA
}

\begin{abstract}
We present ohmage, a mobile to web platform that records, analyzes, and visualizes data from both prompted experience samples entered by the user, as well as continuous streams of data passively collected from sensors onboard the mobile device. ohmage has been used in a number of research health studies. Key challenges in these deployments are engaging participants to sustain data collection in long-lived campaigns, conserving battery power, and extracting accurate inferences from the collected streams. To address these challenges, we have incorporated feedback from hundreds of behavioral and technology researchers, focus group participants, and end-users of the system in an iterative design process. We summarize this rich feedback, and present the resulting system.
\end{abstract}

Keywords- mobile phone, mHealth, activity monitoring

\section{INTRODUCTION}

Mobile phones offer affordable, proximate, personalized, and continuous measurement and interaction, supporting chronic disease prevention and management as part of daily life, 24x7. Engaging individuals through self-monitoring in general has been shown to be critical to succeed in behavioral intervention programs [1], [4]. Leveraging experience sampling (also known as ecological momentary assessment), a technique developed to monitor affect, cognitions, and behavior in real time in a person's natural environment [7], mobile phones can transform self-monitoring into a real-time and interactive process that can react to an individual's context and state.

\section{SYSTEM DESCRIPTION}

\section{A. User feedback to inform system design}

ohmage is an open source smartphone-to-web platform designed to author experience sampling based data collection campaigns in support of a wide range of mobile health pilots and applications [2]. ohmage creates records of behaviors by capturing continuous-autonomous and prompted self-report streams of data for use by researchers and health care providers. ohmage was designed using feedback from hundreds of behavioral and technology researchers, focus group participants [5], and end-users of the system in an iterative design process. An overwhelming majority of people surveyed agreed that customization of reminders and prompt messages is necessary, and that goal setting and feedback are extremely attractive and engaging features. Certain populations were concerned with the burden of completing surveys, and thus prioritized lighter weight methods for recording important events, especially when real-time reporting was necessary.

\section{B. System features}

The following key phone-based features of our platform were designed as a result of the rich user feedback:

- $\quad$ Time and location-triggered self-reports.

- Low-power, automated activity traces including: actigraphy derived from acceleromter, GPS, WiFi, and cell tower radios (Fig. 1); location; exercise; sleep.

- $\quad$ Phone-top buttons for lightweight participant reporting.

- Acoustic traces, which are analyzed to infer social interactions throughout the day.

- Feedback graphics to view past performance at a glance and monitor improvements in measures (see Fig. 2). Using this feature, an individual can compare their current performance with a previously established baseline (calculated on the server). Individuals additionally receive motivational messages based on this baseline comparison.

All data collected from these system components are uploaded to a secure server, where they can be accessed and visualized (e.g. web-based visualization of actigraphy data shown in Fig. 1).

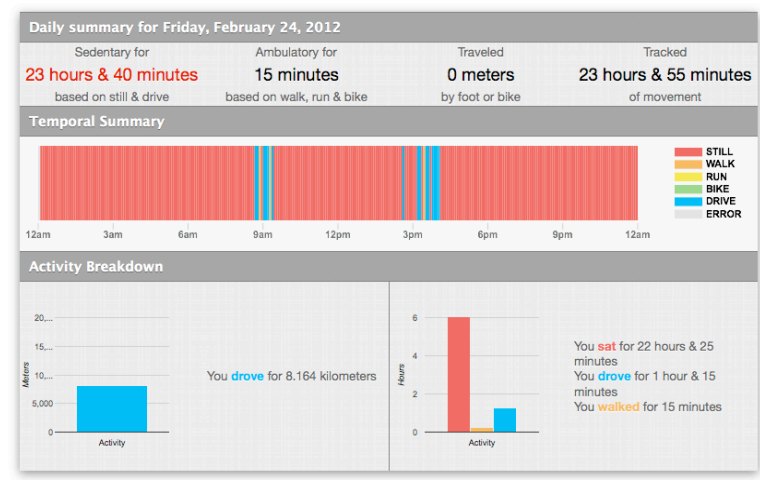

Figure 1. Web-based visualization of actigraphy data streams collected throughout the day. 
Campaign management, data collection authoring enable rapid startup; rich system and user analytics instrument the act of participation itself and aid with interpreting data.

The validity, reliability, usability, and feasibility of ohmage is being evaluated in 7 independent studies, each addressing a different population: recent breast cancer survivors, new moms [6], at-risk HIV+ men, immigrant women [3], young adults with ADHD, individuals managing PTSD, and high school students engaged in data gathering and analysis.

\section{DEMONSTRATION}

We will demonstrate the key features of the phone and server-side ohmage application. In addition, participants with Android phones will be invited to rapidly author and participate in their own campaigns. Data visualizations and feedback graphics will be available on participants' phones and on a website. Participants will be given usernames and passwords to provide secure access to their data. Data collection and graphics will be made available to participants for one month after the conference.



Figure 2. Snapshots of feedback graphics available in the ohmage Android application. Summaries and plots of general mobility states (still, walk, run, drive) captured from the continuous actigraphy trace (top left); graphic comparing baseline responses with responses from current week and past week self-report measures (top right); sparkline time-series and higher resolution time-series plots of various self-report measures (bottom left and right, respectively).

\section{REFERENCES}

[1] Donovan, D. and Marlatt, G. 2005. Assessment of addictive behaviors. Guilford Press.

[2] Hicks, J., Ramanathan, N., Kim, D., Monibi, M., Selsky, J., Hansen, M. \& Estrin, D. AndWellness: An Open Mobile System for Activity and Experience Sampling. In proceedings of Wireless Health 2010, La Jolla, CA.

[3] Khurana, N., Hasnain Wynia, R., Ramanathan, N., Kandula, N. South Asia Women's Perspectives on Mobile Phone Usage and Functionality to Increase Physical Activity. 2012 UIC Minority Health in the Midwest Conference, February 4, 2012. (Oral Presentation.)

[4] Marlatt, G. and Gordon, J. 1985. Relapse Prevention: Maintenance Strategies in Addictive Behavior Change. Guilford Press.

[5] Ramanathan, N., Swendeman, D., Comulada, S., Dawson, B., RotheramBorus, MJ., Estrin, D. Identifying Preferences for Mobile Health Applications Self-Monitoring and Self-Management: Focus Group Findings from HIV-Positive Persons and Young Mothers. Medicine 2.0 2012, Boston, MA. (Oral Presentation)

[6] Selsky, J., Tangmunarunkit, H., Ramanathan, N., Falaki, H., Jenkins, J., Ketcham, C., Longstaff, B., Monibi, M., Ooms, J., Parameswaran, K., Estrin, D. Measures and Real-Time Feedback of Diet, Activity, and Stress Using GPS and Accelerometer Enabled Smartphones. Medicine 2.0 2012, Boston, MA. (Oral Presentation)

[7] Shiffman, S., Stone, A. A., and Hufford, M. R. 2008. Ecological momentary assessment. Annual Review of Clinical Psychology 4, 1, 132. 